\title{
Keppen-Lubinsky syndrome
}

INSERM

\section{Source}

INSERM. (1999). Orphanet: an online rare disease and orphan drug data base. KeppenLubinsky syndrome. ORPHA:435628

A rare, genetic, primary lipodystrophy syndrome characterized by severe developmental delay and intellectual disability, hypertonia, hyperreflexia, microcephaly, tightly adherent skin, an aged appearance, severe generalized lipodystrophy, and distinct facial dysmorphism which includes large prominent eyes, narrow nasal bridge, tented upper lip vermilion, an open mouth, and high-arched palate. Laboratory analysis of serum and urine are normal. 